\title{
A aplicação da aromaterapia como método de alívio da dorem gestantes: uma revisão integrativa
}

\author{
Sandra Cristina de Souza Borges Silva ${ }^{a, *}$, Edymara Tatagiba Medina ${ }^{a}$, \\ Temistocles Barroso de Oliveira ${ }^{\mathrm{b}}$, Simone Sacramento Valverde $^{\mathrm{b}}$ \\ ${ }^{a}$ Faculdade de Enfermagem, Universidade do Estado do Rio de Janeiro, Rio de Janeiro, RJ, Brasil \\ ${ }^{b}$ LaQMed -Laboratório de Química Medicinal de Substâncias Bioativas, Farmanguinhos/FIOCRUZ, Rio de Janeiro,
} RJ, Brasil

Histórico do Artigo

Recebido em:

25/05/2018

Aceito em:

$16 / 07 / 2018$

Palavras-chave:

Cuidados de Enfermagem;

Terapias complementares;

Lavandula angustifolia; Cuidado Pré-natal.

\begin{abstract}
RESUMO
Estudo acerca das intervenções terapêuticas com Aromaterapia, implementadas pela enfermeira em gestantes queixosas de dor. A investigação teve como objetivos identificar a ação analgésica do óleo essencial de lavanda, a ser aplicado em gestantes, assim como descrever os resultados deste cuidado. Trata-se de uma revisão integrativa de literatura, onde utilizou-se a estratégia SPIDER, guiada pela questão norteadora "Quais óleos essenciais possuem ação analgésica durante a gestação?". Por meio de pesquisa nas bases de dados Medical Literature Online (MEDLINE), Literatura Latino-Americana e do Caribe em Ciências da Saúde (LILACS), Bases de Dados de Enfermagem (BDENF), Scientific Electronic Library Online (SciELO), portal da Biblioteca Virtual de Saúde (BVS) e PUBMED, foram selecionados 15 artigos que corresponderam aos objetivos definidos, distribuídos no recorte temporal de 2000 a 2016. Os resultados apontaram a utilização apenas em mulheres durante trabalho de parto e o óleo essencial de lavanda (Lavandula angustifolia) como o mais utilizado, devido às suas ações ansiolítica e analgésica.
\end{abstract}

The application of aromatherapy as a method of patient relief of pain in pregnant: an integrative review
Keywords:

Nursing Care; Complementary

Therapies; Lavandula

angustifolia; Prenatal Care.

\begin{abstract}
Study on therapeutic interventions with Aromatherapy, implemented by the nurse in pregnant complainants of pain. The aim of the investigation was to identify the analgesic action of lavender essential oil use in pregnant women and to describe the results of this application. Integrative literature review, where the SPIDER strategy was used, guided by the guiding question "Which essential oils have analgesic action during gestation?". Through research in the Medical Literature Online (MEDLINE), Latin American and Caribbean Health Sciences Literature (LILACS), Nursing Databases (BDENF), Scientific Electronic Library Online (SciELO), Library portal (VHL) and PUBMED, 15 articles were selected that corresponded to the defined objectives distributed in the temporal cut from 2000 to 2016. The results pointed to the use only in women during labor and lavender essential oil (Lavandula angustifolia) as the most used, due to its anxiolytic and analgesic actions.
\end{abstract}

\section{Introdução}

Desde a década de 80 , os princípios do SUS passaram a orientar as práticas assistenciais em saúde, contemplando o acesso universal e igualitário, a regionalização, a hierarquização e a descentralização dos serviços, o atendimento na perspectiva da integralidade e a participação popular (1). Tais princípios orientadores indicam a necessidade de um modelo assistencial que contemple o indivíduo em sua multidimensionalidade, dentro de uma lógica onde a saúde é vista como o bem-estar nos

* Autor correspondente: scrisborges@ hotmail.com (Silva S.C.S.B.) 
âmbitos biopsicosocioculturais, nos remetendo ao modelo humanizado, onde a autonomia do usuário e a fisiologia do parto são valorizadas.

Tendo como pressupostos as discussões quanto à efetivação do direito à saúde e do acesso aos serviços e aspectos relativos à necessidade de reorganização da atenção básica, das práticas e dos modos de prestar cuidados, os quais são elementos constituintes do modelo assistencial, em meados da década de 90, a Estratégia Saúde da Família (ESF) apresentou-se como o eixo estruturante do processo de reorganização do sistema de saúde, baseado na Atenção Primária à Saúde. As ações nesse âmbito de atenção são dirigidas em acordo com as diretrizes da Política Nacional de Atenção Básica (2), que deslocam o foco do processo assistencial, antes centrado em procedimentos, para um processo centrado no indivíduo, onde o cuidado do usuário é o imperativo ético-político que organiza a intervenção técnico-científica pela equipe multiprofissional.

As intervenções prescritas na ESF configuram um "novo" modelo assistencial, no qual as práticas devem estar orientadas pelos determinantes do processo saúde-doença, considerando o indivíduo no seu contexto familiar, como parte de grupos e de comunidades socioculturais e contemplando ações importantes no campo da Vigilância em Saúde (VS) e da Promoção da Saúde (PS) (3).

O modelo assistencial implementado na atenção básica, pressupõe a utilização de tecnologias assistenciais para a satisfação das necessidades da clientela. Inserida nesse contexto, a enfermeira obstétrica, orientada pelo paradigma humanístico e pelo conceito de desmedicalização, utiliza no processo de cuidar, as tecnologias não invasivas de cuidado de enfermagem obstétrica, definidas como técnicas, procedimentos $\mathrm{e}$ conhecimentos que valorizam a fisiologia e a autonomia femininas (4). Tais ferramentas, aplicadas no cuidado às mulheres durante o ciclo gravídico-puerperal são importantes exemplos de tecnologias leves e leves-duras, na perspectiva da integralidade (5). Dentre elas, destacamos a utilização dos óleos essenciais, fitoterápicos inseridospela Política Nacional de Práticas Integrativas e Complementares (6), na assistência à gestante, diante das queixas de dor durante o trabalho de parto, prática descrita em pesquisas desenvolvidas por enfermeiras.

Durante a gestação, cerca de 50\% das gestantes apresentam queixa de lombalgia (7), conceituada como toda condição de dor ou rigidez, localizada na região inferior do dorso, em uma área situada entre o último arco costal e a prega glútea. Ela ocorre devido a mudanças hormonais; crescimento uterino, distensão de ligamentos uterinos e lombares, promovendo o deslocamento do centro de gravidade da mulher para cima e para frente, podendo acentuar a lordose lombar (8). Embora seja uma queixa frequente, ela ainda é vista como inerente ao período gestacional, sendo pequena a atenção dada a esse sintoma por parte dos profissionais de saúde.

Diante do exposto, surgiu o interesse acerca da utilização do óleo essencial de lavanda, como intervenção terapêutica diante da dor lombar, queixa comum durante o terceiro trimestre gestacional durante os atendimentos pré-natais, objeto dessa pesquisa. Para atender ao objeto proposto, o estudo teve como objetivos identificar a ação analgésica do óleo essencial de lavanda, a ser aplicado em gestantes, assim como descrever os resultados deste cuidado.

\section{Material e métodos}

Estudo descritivo, com abordagem qualitativa e método de revisão integrativa. Tal técnica de pesquisa permite a busca, avaliação e sínteses das evidências disponíveis acerca do tema escolhido; adiciona os resultados referentes à questão de pesquisa; permite indicar lacunas que devem ser preenchidas com novos estudos; reduz as dúvidas quanto às recomendações e fundamenta a prática clínica de qualidade (9). 
Para construir a questão de pesquisa e conduzir as buscas foi utilizada a estratégia SPIDER (10). Essa ferramenta foi desenvolvida a partir de uma adaptação da ferramenta PICO e contempla os seguintes elementos: Sample (amostra); Phenonemon of Interest (fenômeno de interesse); Design (desenho do estudo); Evaluation (avaliações); Research type (tipo de pesquisa). Seu uso permite evidenciar pesquisas com diferentes delineamentos e que abordem determinados comportamentos, relações entre variáveis qualitativas e quantitativas, experiências individuais e coletivas, e intervenções que possuam significados sociais e influenciem na robustez da revisão.

Nesta perspectiva, a busca por artigos, embasada na pergunta "Quais óleos essenciaispossuem ação analgésica durante a gestação?" ocorreu de abril a junho de 2017. Para o desenvolvimento da presente revisão, foram seguidas as etapas: delimitação dos objetivos (PI); busca em bases de dados, ancorada por critérios de inclusão e exclusão (S/D/R); análise crítica dos estudos identificados (E); discussão dos resultados $(\mathrm{E})$; apresentação da revisão.

Os artigos foram selecionados nas bases de dados Medical Literature Online (MEDLINE), Literatura Latino-Americana e do Caribe em Ciências da Saúde (LILACS), Bases de Dados de Enfermagem (BDENF), Scientific Electronic Library Online (SciELO), portal da Biblioteca Virtual de Saúde (BVS) e PUBMED, de março a maio de 2017, utilizando os seguintes termos presentes nos Descritores em Ciências da Saúde (DeCS): gestantes, aromaterapia, analgesia e dor lombar, os quais foram combinados através do operador booleano AND (aromaterapia AND gestante; aromaterapia AND dor lombar; aromaterapia AND analgesia).

Durante a busca, foram utilizados como critério de inclusão: artigos na íntegra nas línguas portuguesa e inglesa, publicados de 2000 a 2016, publicações com metodologias claras sobre o objeto do estudo. Os textos tiveram seu conteúdo avaliado mediante a utilização de instrumento validado (9), que contempla a identificação do artigo original, as suas características metodológicas, além da avaliação dos métodos utilizados, das intervenções apresentadas e dos resultados encontrados.

Para a análise, os artigos foram distribuídos em um quadro sinóptico, que inclui os seguintes dados: nome da pesquisa, nome dos autores, intervenção estudada, resultados e recomendações. A discussão contribuiu para a elucidação dos mecanismos de ação da intervenção estudada, validando sua aplicação nas gestantes.

\section{Resultados}

Inicialmente foram localizados 170 artigos, sendo 26 no PUBMED, 20 no LILACS, 01 no SciELO e 106 na BVS. Após avaliação dos resumos e títulos, foram excluídos 147 estudos, por não atenderem os critérios traçados. Após a leitura completa das 23 investigações restantes, foram selecionadas 15 que atendiam aos objetivos do estudo (Figura 1) 


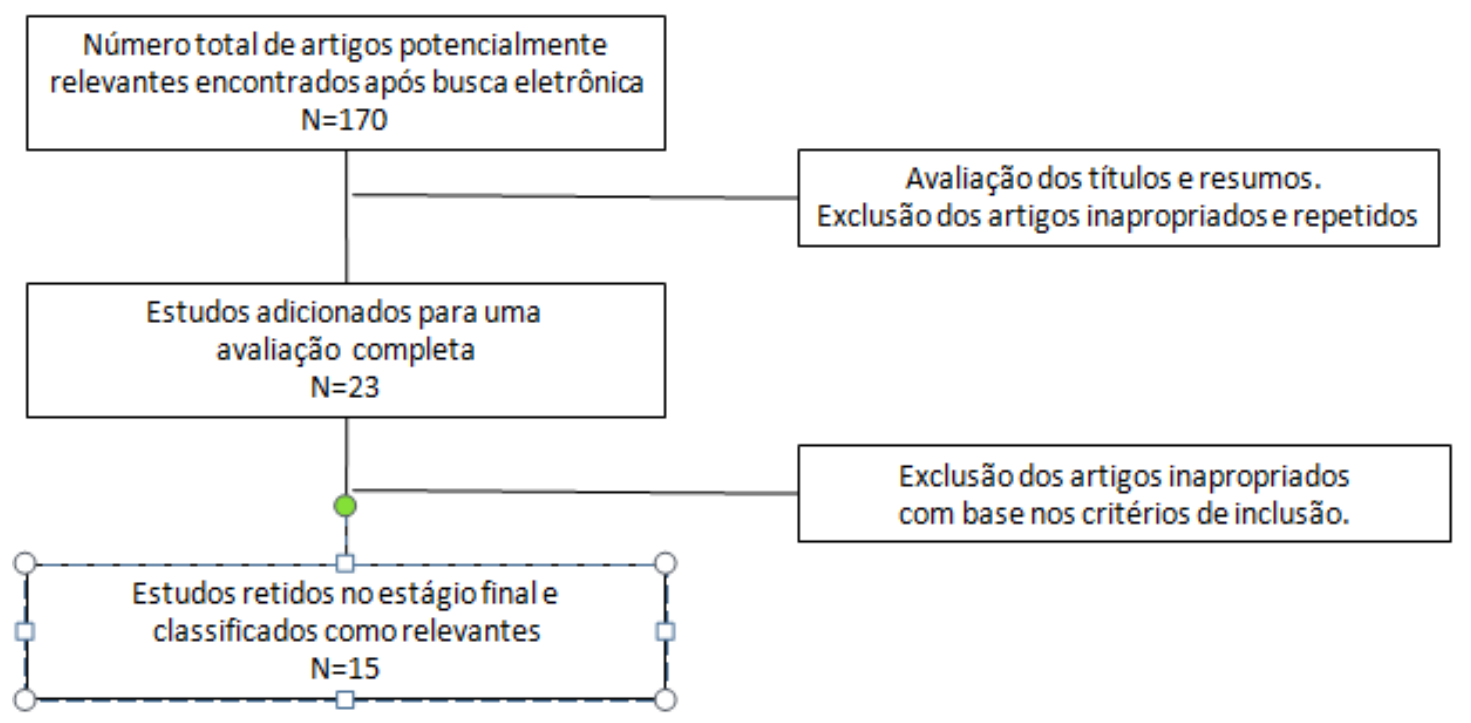

Figura 1 - Representação esquemática do processo de seleção dos artigos.

Dessa amostra, catorze foram publicados originalmente em inglês e um na língua portuguesa. Quanto ao ano de publicação, os estudos foram publicados em 2016 (03), 2013 (04), 2012 (03), 2011 (01), 2010 (02), 2007 (01) e 2000 (01). Oito pesquisas foram desenvolvidas no Irã, três na Inglaterra; e os demais estudos, um foi conduzido na Índia,um na França, um no Brasil e outro nos EUA.

Em relação ao desenho dos estudos, dez tratam-se de ensaios clínicos randomizados (Nível III de evidência científica), três são revisões integrativas (Nível IV de evidência) e duas revisões sistemáticas (Nível I de evidência).

Quadro 1 -Apresentação da síntese de artigos incluídos na revisão integrativa.

\begin{tabular}{|c|c|c|c|c|c|}
\hline Nome do artigo & Idioma/Ano & Autores & $\begin{array}{c}\text { Descritores } \\
\text { utilizados }\end{array}$ & $\begin{array}{c}\text { Intervenção } \\
\text { estudada }\end{array}$ & $\begin{array}{c}\text { Recomendações/ } \\
\text { Conclusões }\end{array}$ \\
\hline $\begin{array}{l}\mathrm{O} \text { impacto da } \\
\text { aromaterapia e } \\
\text { massagem } \\
\text { intraparto sobre o } \\
\text { uso da analgesia e } \\
\text { anestesia em } \\
\text { parturientes;um } \\
\text { caso retrospectivo }\end{array}$ & Inglês/2012 & $\begin{array}{l}\text { Dhany AL, } \\
\text { Mitchell T, Foy } \\
\text { C(30). }\end{array}$ & $\begin{array}{l}\text { Anestesia, } \\
\text { aromaterapia, } \\
\text { massagem,dor,ob } \\
\text { stetrícia/ginecolo } \\
\text { gia }\end{array}$ & $\begin{array}{l}\text { Ensaio clínico } \\
\text { que comparou a } \\
\text { dor na evolução } \\
\text { do trabalho de } \\
\text { parto de } 1.079 \\
\text { parturientes que } \\
\text { receberam } \\
\text { aromaterapia e } \\
\text { masssagem } \\
\text { intraparto, em } \\
\text { comparação com } \\
\text { as informações de } \\
\text { banco de dados } \\
\text { da maternidade. }\end{array}$ & $\begin{array}{l}\text { A utilização da } \\
\text { intervenção está } \\
\text { relacionada à } \\
\text { redução da } \\
\text { utilização de } \\
\text { anestesia } \\
\text { intraparto. }\end{array}$ \\
\hline
\end{tabular}


Vittalle - Revista de Ciências da Saúde v. 31, n. 1 (2019) 61-73

\begin{tabular}{|c|c|c|c|c|c|}
\hline $\begin{array}{l}\text { O manejo da dor } \\
\text { na } \\
\text { parturiente:uma } \\
\text { visão geral das } \\
\text { revisões } \\
\text { sistemáticas }\end{array}$ & Inglês/2013 & Amedee PFJ(31). & Dor, parto & $\begin{array}{l}\text { Revisão } \\
\text { sistemática acerca } \\
\text { dos métodos } \\
\text { farmacológicos e } \\
\text { não } \\
\text { farmacológicos } \\
\text { de alívio da dor } \\
\text { no parto. }\end{array}$ & $\begin{array}{l}\text { A revisão } \\
\text { composta por } 257 \\
\text { estudos aponta que } \\
\text { a massagem pode } \\
\text { prover conforto } \\
\text { nas parturientes. } \\
\text { Quanto à } \\
\text { aromaterapia, são } \\
\text { necessários } \\
\text { estudos para julgar } \\
\text { sua eficácia. }\end{array}$ \\
\hline $\begin{array}{l}\text { Estratégias não } \\
\text { farmacológicas } \\
\text { para alivio da dor } \\
\text { no parto }\end{array}$ & Inglês/2016 & $\begin{array}{l}\text { Hosseni SF, } \\
\text { PilevarzadehM,V } \\
\text { azirinasab H(32). }\end{array}$ & $\begin{array}{l}\text { Alivio da } \\
\text { dor,parto,métodos } \\
\text { não } \\
\text { farmacológicos }\end{array}$ & $\begin{array}{l}\text { Ensaio clinico } \\
\text { com aplicação de } \\
\text { massagem, } \\
\text { aromaterapia e } \\
\text { exercicios } \\
\text { respiratórios para } \\
\text { alívio da dor em } \\
308 \text { parturientes. }\end{array}$ & $\begin{array}{l}\text { Em } 26 \% \text { das } \\
\text { parturientes, a } \\
\text { aromaterapia foi } \\
\text { uma intervenção } \\
\text { efetiva na } \\
\text { redução da dor. }\end{array}$ \\
\hline $\begin{array}{l}\text { Abordagens } \\
\text { alternativas e } \\
\text { complementares } \\
\text { para alivio da dor } \\
\text { durante o parto }\end{array}$ & Inglês/2013 & $\begin{array}{l}\text { Angel RG,Sriram } \\
\text { N, Soli,TK(33). }\end{array}$ & $\begin{array}{l}\text { Relaxamento, } \\
\text { hidroterapia,musi } \\
\text { coterapia,meditaç } \\
\text { ão, acupuntura }\end{array}$ & $\begin{array}{l}\text { Revisão } \\
\text { integrativa acerca } \\
\text { dos métodos não } \\
\text { farmacológicos } \\
\text { de alívio da dor } \\
\text { no parto. }\end{array}$ & $\begin{array}{l}\text { As terapias } \\
\text { alternativas e } \\
\text { complementares } \\
\text { são usadas diante } \\
\text { da ineficácia dos } \\
\text { métodos } \\
\text { tradicionais para } \\
\text { alivio da dor no } \\
\text { parto e satisfazer } \\
\text { as necessidades } \\
\text { da parturiente. }\end{array}$ \\
\hline $\begin{array}{l}\text { Aromaterapia } \\
\text { nonascimento:um } \\
\text { estudo piloto } \\
\text { controlado } \\
\text { randomizado }\end{array}$ & Inglês/2007 & $\begin{array}{l}\text { Burns E, Zobbi } \\
\text { V, Panzeri D, } \\
\text { Oskrochi } \\
\text { R,Regalia A(34). }\end{array}$ & $\begin{array}{l}\text { Aromaterapia, } \\
\text { parto }\end{array}$ & $\begin{array}{l}\text { Ensaio clinico com } \\
\text { aplicação da } \\
\text { aromaterapia } \\
\text { como estratégia } \\
\text { para melhorar os } \\
\text { resultados materno } \\
\text { e neonatais. }\end{array}$ & $\begin{array}{l}\text { Foi identificado } \\
\text { alivio da dor em } \\
\text { primíparas. Não } \\
\text { ocorreu diferença } \\
\text { significativa nos } \\
\text { desfechos } \\
\text { relacionados a } \\
\text { indicação de } \\
\text { cesariana, uso de } \\
\text { Kristeler ou } \\
\text { duração do } \\
\text { primeiro e } \\
\text { segundo estágios } \\
\text { do trabalho de } \\
\text { parto. }\end{array}$ \\
\hline $\begin{array}{l}\text { Analgesia não } \\
\text { farmacológica } \\
\text { no parto }\end{array}$ & Inglês/2013 & $\begin{array}{l}\text { Arendt KW, } \\
\text { Tessmer-Tuck } \\
\text { JA(35). }\end{array}$ & $\begin{array}{l}\text { Hidroterapia, } \\
\text { Acupuntura,TEN } \\
\text { S,yoga,hipnose } \\
\text { aromaterapia, } \\
\text { pápulas de água } \\
\text { estéril }\end{array}$ & $\begin{array}{l}\text { Revisão } \\
\text { integrativa acerca } \\
\text { da Hidroterapia, } \\
\text { Acupuntura,TEN } \\
\text { S, yoga,hipnose } \\
\text { aromaterapia, } \\
\text { pápulas de água } \\
\text { estéril. }\end{array}$ & $\begin{array}{l}\text { A aplicação do } \\
\text { óleo essencial de } \\
\text { lavanda através } \\
\text { do banho de } \\
\text { imersão, } \\
\text { aplicação tópica } \\
\text { ou inalação é uma } \\
\text { intervenção } \\
\text { benéfica. }\end{array}$ \\
\hline
\end{tabular}


S. C. S. B. Silva et al./Vittalle v. 31, n. 1 (2019) 61-73

\begin{tabular}{|c|c|c|c|c|c|}
\hline $\begin{array}{l}\text { Métodos não } \\
\text { farmacológicos } \\
\text { para alívio da dor } \\
\text { no trabalho de } \\
\text { parto: uma } \\
\text { revisão } \\
\text { sistemática }\end{array}$ & $\begin{array}{l}\text { Português/ } \\
2010\end{array}$ & $\begin{array}{l}\text { Gayeski ME, } \\
\text { Brüggemann } \\
\text { OM(36) }\end{array}$ & $\begin{array}{l}\text { Trabalho de } \\
\text { parto. Dor do } \\
\text { parto. Ensaio } \\
\text { clínico. } \\
\text { Enfermagem } \\
\text { obstétrica }\end{array}$ & $\begin{array}{l}\text { Revisão } \\
\text { integrativa acerca } \\
\text { dos métodos não } \\
\text { farmacológicos de } \\
\text { alivio da dor, no } \\
\text { trabalho de parto. }\end{array}$ & $\begin{array}{l}\text { A dor, a } \\
\text { ansiedade e } \\
\text { omedo foram } \\
\text { menores para } \\
86 \% \text { das } \\
\text { mulheres } \\
\text { quereceberam a } \\
\text { intervenção. Em } \\
\text { relação aos } \\
\text { resultadosneonata } \\
\text { is, houve uma } \\
\text { redução nas } \\
\text { transferências de } \\
\text { neonatos para a } \\
\text { terapia intensiva. }\end{array}$ \\
\hline $\begin{array}{l}\text { O uso inalatório } \\
\text { da lavanda na dor } \\
\text { do parto }\end{array}$ & Inglês/2012 & $\begin{array}{l}\text { Vakilian } \\
\text { K,Karamat A, } \\
\text { Mousavi A, } \\
\text { Shariati M, Ajani } \\
\text { E, Atarha M(37) }\end{array}$ & $\begin{array}{l}\text { Aromaterapia, } \\
\text { medicina } \\
\text { complementar, } \\
\text { lavanda, dor }\end{array}$ & $\begin{array}{l}\text { Ensaio clínico, } \\
\text { com amostra de } \\
120 \text { mulheres, } \\
\text { que fizeram uso } \\
\text { inalatório do } \\
\text { vapor frio de óleo } \\
\text { essencial de } \\
\text { lavanda, para } \\
\text { alivio da dor no } \\
\text { parto. O grupo } \\
\text { controle usou } \\
\text { vapor frio de } \\
\text { água. A } \\
\text { intensidade da } \\
\text { dor foi avaliada } \\
\text { pela escala de } \\
\text { analogia visual. }\end{array}$ & $\begin{array}{l}\text { O grupo que } \\
\text { recebeu a lavanda } \\
\text { por via inalatória } \\
\text { relatou alívio da } \\
\text { dor no parto. }\end{array}$ \\
\hline $\begin{array}{l}\text { O efeito da } \\
\text { aromaterapia com } \\
\text { lavanda na } \\
\text { intensidadee } \\
\text { percepção da dor } \\
\text { intraparto em } \\
\text { primíparas }\end{array}$ & Inglês/2010 & $\begin{array}{l}\text { Alavi N, Nemati } \\
\text { M, Kaviani M, } \\
\text { Tabaie M(38). }\end{array}$ & $\begin{array}{l}\text { Aromaterapia, } \\
\text { lavanda,trabalho } \\
\text { de } \\
\text { parto,primípara }\end{array}$ & $\begin{array}{l}\text { Ensaio clínico, } \\
\text { com amostra de } \\
160 \text { parturientes } \\
\text { que receberam o } \\
\text { óleo essencial de } \\
\text { lavanda por via } \\
\text { inalatoria, } \\
\text { enquanto o grupo } \\
\text { controle recebeu } \\
\text { água destilada } \\
\text { pela mesma via. } \\
\text { A intensidade da } \\
\text { dor foi avaliada } \\
\text { pela escala de } \\
\text { analogia visual. }\end{array}$ & $\begin{array}{l}\text { A análise revelou } \\
\text { que não } \\
\text { hádiferença } \\
\text { significativa na } \\
\text { percepção } \\
\text { dolorosa entre os } \\
\text { dois grupos. No } \\
\text { grupo que recebeu } \\
\text { a lavanda a dor for } \\
\text { menos intensa } 30 \\
\text { e } 60 \text { minutos após } \\
\text { a intervenção. }\end{array}$ \\
\hline
\end{tabular}


Vittalle - Revista de Ciências da Saúde v. 31, n. 1 (2019) 61-73

\begin{tabular}{|c|c|c|c|c|c|}
\hline $\begin{array}{l}\text { Comparação } \\
\text { entre os efeitos da } \\
\text { aromaterapia com } \\
\text { lavanda e as } \\
\text { técnicas de } \\
\text { respiração na } \\
\text { redução da dor } \\
\text { durante o trabalho } \\
\text { de parto. }\end{array}$ & Inglês/2011 & $\begin{array}{l}\text { Seraji A, Vakilian } \\
\mathrm{K}(39) \text {. }\end{array}$ & $\begin{array}{l}\text { Aromaterapia, } \\
\text { medicina } \\
\text { complementar, } \\
\text { tecnicas de } \\
\text { respiração, dorno } \\
\text { trabalho de parto, } \\
\text { lavanda }\end{array}$ & $\begin{array}{l}\text { Ensaio clinico } \\
\text { onde foi } \\
\text { administrado o } \\
\text { óleo essencial de } \\
\text { lavanda por via } \\
\text { inalatoria em } 60 \\
\text { parturientes } \\
\text { durante a fase } \\
\text { ativa do trabalho } \\
\text { de parto, o grupo } \\
\text { controle recebeu } \\
\text { água destilada. } \\
\text { Ambos os grupos } \\
\text { realizaram } \\
\text { técnicas } \\
\text { respiratórias. A } \\
\text { intensidade da } \\
\text { dor foi avaliada } \\
\text { pela escala de } \\
\text { analogia visual. }\end{array}$ & $\begin{array}{l}\text { Os resultados } \\
\text { indicaram } \\
\text { melhores } \\
\text { respostas ao } \\
\text { escore de dor nas } \\
\text { parturientes que } \\
\text { inalaram a } \\
\text { lavanda. }\end{array}$ \\
\hline $\begin{array}{l}\text { Massagens e } \\
\text { aromaterapia com } \\
\text { lavanda na } \\
\text { redução da dor e } \\
\text { da duração do } \\
\text { trabalho de parto: } \\
\text { Caso controle } \\
\text { randomizado. }\end{array}$ & Inglês/2013 & $\begin{array}{l}\text { ZahraA,Mohamm } \\
\text { adkhani SL(40). }\end{array}$ & $\begin{array}{l}\text { Aromaterapia, } \\
\text { métodos } \\
\text { complementares/a } \\
\text { lternativos,dor no } \\
\text { trabalho de } \\
\text { parto,massagem, } \\
\text { óleo de lavanda }\end{array}$ & $\begin{array}{l}\text { Ensaio clinico } \\
\text { onde } 60 \text { mulheres } \\
\text { foram } \\
\text { distribuídas em } \\
\text { dois grupos } \\
\text { iguais, metade } \\
\text { recebeu } \\
\text { massagem e a } \\
\text { outra metade } \\
\text { massagem com } \\
\text { uso do óleo } \\
\text { essencial de } \\
\text { lavanda.A } \\
\text { intensidade da } \\
\text { dor foi avaliada } \\
\text { pela escala de } \\
\text { analogia visual. }\end{array}$ & $\begin{array}{l}\text { A dor foi menos } \\
\text { intensa nas } \\
\text { parturientes que } \\
\text { receberam } \\
\text { massagem e óleo } \\
\text { de lavanda, } \\
\text { durante as fases } \\
\text { passiva e ativa do } \\
\text { trabalho de parto. } \\
\text { Elas também } \\
\text { tiveram o } \\
\text { primeiro e o } \\
\text { segundo estágios } \\
\text { do trabalho de } \\
\text { parto mais curtos. }\end{array}$ \\
\hline
\end{tabular}


S. C. S. B. Silva et al./Vittalle v. 31, n. 1 (2019) 61-73

\begin{tabular}{|c|c|c|c|c|c|}
\hline $\begin{array}{l}\text { Uma investigação } \\
\text { acerca do uso da } \\
\text { aromaterapia } \\
\text { intraparto, na } \\
\text { prática das } \\
\text { midwifes(obstetri } \\
\text { zes) }\end{array}$ & Inglês/2000 & $\begin{array}{l}\text { Burns EE, } \\
\text { Blamey C, Ersser } \\
\text { SJ, Barnetson L, } \\
\text { Lloyd AJ(41) }\end{array}$ & Aromaterapia, dor & $\begin{array}{l}\text { Estudo } \\
\text { prospectivo que } \\
\text { avaliou a ação da } \\
\text { aromaterapia em } \\
8058 \text { gestantes } \\
\text { atendidas em } \\
\text { maternidade de } \\
\text { hospital de } \\
\text { ensino, de } 1990 \text { a } \\
\text { 1998, com o } \\
\text { objetivo de } \\
\text { verificar o alívio } \\
\text { da dor. }\end{array}$ & $\begin{array}{l}\text { O uso da } \\
\text { aromaterapia } \\
\text { reduziu a } \\
\text { necessidade da } \\
\text { utilização de } \\
\text { outrosanalgésicos } \\
\text {, Durante o } \\
\text { período estudado, } \\
\text { nessa unidade } \\
\text { ocorreu uma } \\
\text { redução no uso de } \\
\text { petidina nas } \\
\text { parturientes, de } \\
6 \% \text { para } 0,2 \% \text {. O } \\
\text { estudo também } \\
\text { demonstrou que a } \\
\text { aromaterapia } \\
\text { pode ter a ação de } \\
\text { potencializar as } \\
\text { contrações } \\
\text { ineficazes. }\end{array}$ \\
\hline $\begin{array}{l}\text { O efeito da } \\
\text { massagem com } \\
\text { óleo essencial de } \\
\text { lavanda na } \\
\text { intensidade da } \\
\text { dor durante a fase } \\
\text { ativa e na } \\
\text { satisfação no } \\
\text { trabalho de parto } \\
\text { em nulíparas. }\end{array}$ & Inglês 2012 & $\begin{array}{l}\text { Mohammadkhani } \\
\text { SL,AbbaspoorZ, } \\
\text { Aghel } \\
\text { N,Mohammadkha } \\
\text { ni SH(42). }\end{array}$ & $\begin{array}{l}\text { Aromaterapia, dor } \\
\text { no parto,primípara }\end{array}$ & $\begin{array}{l}\text { Ensaio clínico } \\
\text { onde } \\
\text { 90parturientes } \\
\text { foram divididas } \\
\text { em } 3 \text { grupos } \\
\text { iguais que } \\
\text { receberam } 20 \\
\text { minutos de } \\
\text { massagem em } \\
\text { região lombar. O } \\
\text { primeiro recebeu } \\
\text { massagem, o } \\
\text { segundo recebeu } \\
\text { massagem com } \\
\text { óleo de amêndoa } \\
\text { e o terceiro } \\
\text { recebeumassagem } \\
\text { com óleo } \\
\text { essencial de } \\
\text { lavanda. A } \\
\text { intensidade da } \\
\text { dor foi mensurada } \\
\text { pela escala de } \\
\text { analogia visual } \\
\text { antes e depois da } \\
\text { intervenção. }\end{array}$ & $\begin{array}{l}\text { A dor foi menos } \\
\text { intensa no grupo } \\
\text { que } \\
\text { recebeumassagem } \\
\text { com óleo essencial } \\
\text { de lavanda. }\end{array}$ \\
\hline $\begin{array}{l}\text { Revisão de } \\
\text { ensaios clínicos } \\
\text { randomizados } \\
\text { sobre os efeitos } \\
\text { do óleo essencial } \\
\text { de lavanda na dor } \\
\text { durante o trabalho } \\
\text { de parto }\end{array}$ & Inglês/2016 & $\begin{array}{l}\text { Makvandi S, } \\
\text { Mirteimoori M, } \\
\text { Najmabadi } \\
\text { KM(43). }\end{array}$ & $\begin{array}{l}\text { Aromaterapia, } \\
\text { lavanda, } \\
\text { nascimento, } \\
\text { manejo da dor, } \\
\text { análise estatística }\end{array}$ & $\begin{array}{l}\text { Revisão } \\
\text { sistemática e } \\
\text { metanálise. }\end{array}$ & $\begin{array}{l}\text { A Aromaterapia } \\
\text { com OE lavanda } \\
\text { por inalação } \\
\text { diminuiu a dor de } \\
\text { parto, assim } \\
\text { como a } \\
\text { massagem } \\
\text { aromaterápica. }\end{array}$ \\
\hline
\end{tabular}




\begin{tabular}{|c|c|c|c|c|c|}
\hline $\begin{array}{l}\text { O efeito do óleo } \\
\text { essencial de } \\
\text { lavanda da dor e } \\
\text { na duração do } \\
\text { trabalho de parto } \\
\text { em primíparas. }\end{array}$ & Inglês/2016 & $\begin{array}{l}\text { Yazdkhasti M; } \\
\text { Pirak A(44). }\end{array}$ & $\begin{array}{l}\text { Trabalho de } \\
\text { parto, óleo } \\
\text { essencial de } \\
\text { lavanda, } \\
\text { primípara, } \\
\text { Aromaterapia }\end{array}$ & $\begin{array}{l}\text { Ensaio } \\
\text { clínicorandomiza } \\
\text { do, foirealizado } \\
\text { em 120gestantes } \\
\text { em dois grupos. } \\
\text { O grupo } \\
\text { experimental } \\
\text { recebeu } 2 \text { gotas } \\
\text { de essência } \\
\text { delavanda inalada } \\
\text { em três estágios } \\
\text { (4-5, 6-7, 8-9cm } \\
\text { de dilatação } \\
\text { cervical) e a } \\
\text { intensidade da } \\
\text { dor de parto e a } \\
\text { duração do } \\
\text { trabalho foi } \\
\text { medida antes e } \\
\text { após a } \\
\text { intervenção. O } \\
\text { grupo controle foi } \\
\text { tratado comágua } \\
\text { destilada como } \\
\text { placebo de } \\
\text { maneira similar }\end{array}$ & $\begin{array}{l}\text { Os } \\
\text { resultadosmostrar } \\
\text { am que } \\
\text { adiferença na dor } \\
\text { doparto antes e } \\
\text { após aintervenção } \\
\text { em dois grupos } \\
\text { foisignificativa (P } \\
\text { 1/40/001). Mas } \\
\text { nãohouve } \\
\text { diferença } \\
\text { naduração média } \\
\text { dafase ativa e } \\
\text { nosegundo } \\
\text { estágio } \\
\text { detrabalho entre } \\
\text { osdois grupos }\end{array}$ \\
\hline
\end{tabular}

Verificou-se que os artigos incluídos nesta revisão estudaram o uso da aromaterapia em parturientes. Não foram identificados estudos que tratassem das queixas de dor em outro momento da gestação, o que pode indicar uma tendência de pouca valorização dos desconfortos comuns durante a gravidez, como a lombalgia, a cefaleia, dores em membros inferiores, por exemplo; sugerindo que tais queixas possam ser tratadas de modo tradicional, por meio da indicação de analgésicos - apesar de existirem outros métodos, como alongamentos, massagens e exercícios descritos por Janet Balaskas (11); porém são pouco explorados nos estudos acadêmicos.

Uma vez que todas as pesquisas tratam de intervenções indicadas à dor no nascimento em cenário hospitalar ou domiciliar, tal movimento também sinaliza que, no universo estudado, a queixa de dor não foi contemplada como necessidade prioritária no cuidado pré-natal, apesar de frequente na população geral de gestantes (12-13). Os estudos encontrados tratam a dor como evento biológiconegativo e restrito ao trabalho de parto e parto;aspecto esse que reforça o nascimento como sinônimo de sofrimento maternoe o profissional que assiste à mulher se vê na obrigação de cessá-lo; fazendo referência ao modelo tecnocrático, onde a mulher é vítima da sua própria natureza (14) e não tem seu protagonismo e fisiologia valorizados.

Em contraponto, foi salientado que os objetivos e resultados dos ensaios clínicos presentes na amostra apontam a preocupação dos pesquisadores em verificar a ação dos óleos essenciais como agentes que favorecem o alivio da dor. Seis dos quinze estudos indicam a Aromaterapia como intervenção favorável nesse sentido, sem nomear especificamente o óleo essencial administrado. Justificamos essa observação devido à tal parcela de pesquisas incluir as revisões sistemáticas e integrativas localizadas e pelo fato de estas publicações tratarem dos efeitos da Aromaterapia no geral, sendo apontada como uma das Práticas Integrativas e Complementares existentes em diversas localidades do mundo, sobretudo no Oriente Médio - onde ela faz parte da cultura milenar.

Sete ensaios clínicos randomizados, pertencentes à amostra, encontraram relações 
entre a administração inalatória ou por via tópica do óleo essencial de lavanda (Lavandulaangustifolia) e o alívio da dor durante o trabalho de parto e parto. Alguns destes trabalhos correlacionaram esta intervenção à abreviação do primeiro e do segundo períodos clínicos do parto. A ansiedade, outra queixa comum das gestantes $(15,16)$ foi também abordada em pelo menos três estudos, onde a administração do óleo essencial de lavanda resultou em diminuição significativa deste sintoma.

A caracterização química do óleo essencial de lavanda indica que os seus compostos majoritários são linalol e acetato de linalila (17); elementos com ações antinociceptiva, citofilática, anti-inflamatória, ansiolítica e sedativa (18); o que justifica os efeitos encontrados nas amostras dos estudos realizados.

No que se refere à atenção à mulher no ciclo gravídico-puerperal, o modelo assistencial excessivamente biomédico vem sendo criticado mundialmente; culminando na avaliação sistemática destas práticas obstétricas(19). Os resultados iniciais, ainda na década de 1990, deram origem a diretrizes e recomendações com base em evidências científicas, que a Organização Mundial de Saúde e o Ministério da Saúde $(19,20)$ vêm adotando ao longo dosúltimos anos. Tais bases consideram a gestação e o parto como eventos fisiológicos que necessitam de apoio, avaliação e vigilância, onde as intervenções devem ser justificadas edecididas em conjunto com a mulher $(19,20)$. Nessa perspectiva, é viabilizada a desmedicalização da assistência, na qual a equipe multiprofissional apoia as escolhas da mulher acerca dos cuidados.

No Brasil, a transformação das práticas de assistência obstétrica nos âmbitos da Atenção Básica e Hospitalar vem acontecendo paulatinamente desde 1990, a partir de políticas públicas baseadas no cuidado respeitoso e na promoção do protagonismo feminino. Dentre as medidas, há a recomendação deque enfermeiras obstétricas promovam o cuidado à gestante de baixo risco, por apresentar vantagens no que se refere à atenção focada na fisiologia feminina e na prevenção de intervenções realizadas sem critério algum $(21,22)$.Estes resultados se devem à disposição dessas profissionais em desenvolver habilidades específicas, num modelo embasado nos pressupostos da humanização (23) e produzindo conhecimento centrado no protagonismo feminino (24-26).Nesse sentido, a enfermeira obstétrica utiliza tecnologias de cuidado denominadas não invasivas,pois nesse contexto são priorizadas a fisiologia e a autonomia femininas (26).

Portanto, neste contexto colocado, a Aromaterapia se mostra como uma prática melhor aceita entre as enfermeiras obstétricas - devido ao fato de não exigir grande espaço ou equipamentos sofisticados para realizá-la, necessitando apenas de óleos essenciais adequados para cada situação - e pelas clientes, por conta do odor agradável e da sensação de bem-estar e cuidado - proporcionado tanto pela ação terapêutica dos óleos essenciais como também do cuidado empático e acolhedor, promovido pela enfermeira obstétrica $(26,27)$.

Os princípios humanistas e holísticos encontramconfluência com as práticas integrativas como a Aromaterapia, contribuindo para a ampliação de cuidados prestados no sentido de oferecer uma abordagem que não somente almeje o bem-estar físico, mas também mental, emocional e espiritual das mulheres. A Aromaterapia, historicamente, tem sido praticada por diversos profissionais de saúde, dentre eles, os enfermeiros. Há registros de que Florence Nightingale, durante a Guerra da Crimeia, aplicava óleo essencial de Lavanda na fronte dos soldados feridos com o intuito de promover tranquilidade (28). Os enfermeiros são pioneiros na utilização de Práticas Integrativas e Complementares; possuem respaldo em todo o território brasileiro através de portarias do Ministério da Saúde, que regulamenta as Práticas, e do próprio Conselho Federal de Enfermagem $(6,29)$. 


\section{Considerações Finais}

Nesta revisão integrativa foram discutidosartigos que abordaram a Aromaterapia como estratégia para o alivio da dor em gestantes. Elesapontaram a eficácia desta, sendo o óleo essencial de lavanda (Lavandula angutifolia) o mais utilizado no alívio da dor durante otrabalho de parto, assim como na redução da ansiedade. Também foram encontradas relações entre a administração desse agente e o encurtamento da fase ativa do trabalho de parto; que se justificam pelos elementos químicos preponderantes no óleo essencial de lavanda - o linalol e o acetato de linalila - parecem favorecer a ação dos hormônios femininos presentes no trabalho de parto e parto, através da supressão do neocórtex e ação do "cérebro primitivo"; ou seja, o óleo essencial de lavanda favorece concentração e tranquilidade à mulher.

Não foram encontrados estudos que abordassem especificamente a Aromaterapia aplicada às gestantes queixosas por dor lombar fora do trabalho de parto; entretanto, o uso durante a gestação deveria ser recomendado por aliar em um mesmo procedimento, duas ações importantíssimas: a ação antinociceptora (reduz a dor lombar) e ansiolítica principalmente no final da gestação. Outro benefício seria a inclusão de um familiar no cuidado à gestante através da massagem com o óleo essencial, favorecendo o vínculo e também se beneficiando com as ações terapêuticas do óleo. Existem algumas maternidades públicas, incentivadas pelos programas de Residência em Enfermagem Obstétrica - como é o caso do município do Rio de Janeiro - e Casas de Parto existentes no Brasil; que realizam este trabalho com as gestantes e suas famílias e há resultados bem expressivos e importantes, tanto para a consolidação do trabalho da enfermeira obstétrica quanto para a construção de políticas públicas que colocam a mulher como protagonista do cuidado.

Porém, apesar das recomendações quanto à implementação das Práticas Integrativas e Complementares nos serviços públicos de saúde e de haver estudos mostrando os benefícios da Aromaterapia - em especial o óleo essencial de Lavandula angustifoliano alívio de dor em gestantes; ainda faltam estudos e incentivos para novas pesquisas, a fim de estabelecer níveis de evidência mais fortes e assim, validar um conhecimento que é, além de científico, milenar em diversas culturas.

Sugerimos, portanto, a realização de novas pesquisas, sobretudoensaios clinicos randomizados acerca da temática e que esta possa ser melhor explorada nos cursos de graduação e pós-graduação em Saúde, como forma de qualificar o trabalho e incentivar a produção científica brasileira.

\section{Referências}

1. Fertonani HP, Pires DEP, Biff D, Scherer MDA. Modelo assistencial em saúde: conceitos e desafios para a atenção básica brasileira. Ciênc Saúde Colet 2015; 20(6):1869-78.

2. Brasil. Ministério da Saúde. Portaria $n^{\circ} 2.488$, de 21 de outubro de 2011. Aprova a Política Nacional de Atenção Básica, estabelecendo a revisão de diretrizes e normas para a organização da atenção básica, para a Estratégia Saúde da Família (ESF) e o Programa de Agentes Comunitários de Saúde (PACS)[Internet]. Brasília: Ministério da Saúde [citado em 2018 Abr 30]. Disponível em: http://www.saude.mt.gov.br/upload/legislacao/2488-[5046-041111-SES-MT].pdf

3. Brasil. Ministério da Saúde (MS). Secretaria de Assistênciaà Saúde. Coordenação de Saúde da Comunidade. Saúde da Família: uma estratégia para reorientação do modelo assistencial. Brasília: MS; [página na internet] 1997. [acessado 2018abr 10]. Disponível em: http://bvsms.saude.gov.br/bvs/publicacoes/cd09_16.pdf

4. Seibert SL. Tecnologias não-invasivas de cuidado de enfermagem obstétrica no suporte físico à parturiente: critérios e efeitos esperados [Dissertação de mestrado].Rio de Janeiro: Universidade Estadual do Rio de Janeiro; 2010. 
5. Merhy EE. Em busca de ferramentas analisadoras das Tecnologias em Saúde: a informação e o dia a dia de um serviço, interrogando e gerindo trabalho em saúde.In:Merhy EE, Onocko R, editores. Agir em saúde: um desafio para o público. $2^{\mathrm{a}}$ ed. São Paulo: Hucitec; 2002. p. 113-50.

6. Brasil. Ministério da Saúde. Portaria $n^{\circ}$ 849, de 27 de março de 2017. Inclui a Arteterapia, Ayurveda, Biodança, Dança Circular, Meditação, Musicoterapia, Naturopatia, Osteopatia, Quiropraxia, Reflexoterapia, Reiki, Shantala, Terapia Comunitária Integrativa e Yoga à Política Nacional de Práticas Integrativas e Complementares. [Internet]. Brasília: Ministério da Saúde [citado em 2018 Abr 30 ]. Disponível em: https://www.jusbrasil.com.br/diarios/141355541/dou-secao-1-28-03-2017-pg-68/pdf

7. FerreiraHC, Nakano AMS. Reflexões sobre as bases conceituais que fundamentam a construção do conhecimento acerca da lombalgia na gestação. Rev latinoam enferm 2001; 9(3): 95-100.

8. Mann L, Kleinpaul JF, Teixeira CS, Konopka CK. Dor lombo-pélvica e exercício físico durante a gestação. Fisioter mov 2008;21(2):99-105.

9. Mendes K, Silveira R, Galvão CM. Revisão integrativa: método de pesquisa para a incorporação de evidências na saúde e na enfermagem. Texto\&context enferm 2008; 17(4): 758-64.

10. Cooke A, Smith D, Booth A. Beyond PICO: the SPIDER tool for qualitative evidence synthesis. Qual health res 2012; 22(10):1435-43.

11. Balaskas J. Parto ativo: Guia prático para o parto natural - A história e a filosofia de uma revolução. $3^{\mathrm{a}}$ ed. São Paulo: Editora Ground; 2016.

12. Ursi ES, Galvão CM. Prevenção de lesões de pele no perioperatório: revisão integrativa da literatura. Revlatinoamenferm 2006; 14(1):124-31.

13. Santos MMD, Gallo AP. Lombalgia gestacional: prevalência e características de um programa prénatal. Arqbrasciênc saúde 2010; 35(3):174-9.

14. Diniz CSG. Humanização da assistência ao parto no Brasil: os muitos sentidos de um movimento. Ciênc Saúde Colet 2005;10(3):627-37.

15. Araújo DMR, Pacheco AHRN, Pimenta AM, Kac G. Prevalência e fatores associados a sintomas de ansiedade em uma coorte de gestantes atendidas em um centro de saúde do município do Rio de Janeiro. Rev bras saúde matern infant 2008; 8(3):333-40.

16. Schetter CD, Tanner L. Anxiety, depression and stress in pregnancy: implications for mothers, children, research, and practice. Curr opin psychiatr 2012;25(2):141-8.

17. Machado MP, Ciotta MN, Deschamps C, Zanette F, Côcco LC, Biasi LA. Propagação in vitro e caracterização química do óleo essencial de Lavandula angustifolia cultivada no Sul do Brasil. Ciênc rural 2013; 43(2):283-9.

18. Camargo SB, Vasconcelos DFSA. Atividades biológicas de Linalol: conceitos atuais e possibilidades futuras deste monoterpeno. Rev Ciênc Méd Biol 2015;13(3):381-7.

19. World Health Organization.WHO recommendations:intrapartum care for a positive childbirth experience. Geneva: World Health Organization; 2018. Licence: CC BY-NC-SA 3.0 IGO.

20. Brasil. Ministério da Saúde. Diretrizes Nacionais de Assistência ao Parto Normal. [Internet]. Brasília: Ministério da Saúde [citado em 2018 Abr 20]. Disponível em: http://portalarquivos.saude.gov.br/images/pdf/2017/marco/08/Diretrizes-Parto-Normal-resumidaFINAL.pdf

21. National Collaborating Centre for Women's and Children's Health (Great Britain). Intrapartum care: care of healthy women and their babies during childbirth. National Institute for Health and Care Excellence (NICE). 2014. [citadoem 2018 Abr 20]. Disponívelem: https://www.nice.org.uk/guidance/cg190

22. Vargens OMC, Progianti JM, Silveira ACF. O significado de desmedicalização da assistência ao parto no hospital: análise da concepção de enfermeiras obstétricas. Rev Esc Enferm USP 2008; 42(2):339-46.

23. Gomes ML, Moura MAV, Souza IEO. A prática obstétrica da enfermeira no parto institucionalizado: uma possibilidade de conhecimento emancipatório. Texto\&context enferm 2013;22(3):763-71.

24. Keating A, Fleming VEM. Midwives' experiences of facilitating normal birth in an obstetric-led unit: a feminist perspective. J obstet gynecol neonatal nurs. 2009;25(5):518-27.

25. Kennedy HP, Shannon MT. Keeping Birth Normal: Research Findings on Midwifery Care During Childbirth. J obstet gynecol neonatal nurs 2004; 33(5):554-60.

26. Progianti JM, Vargens OMC. As enfermeiras obstétricas frente ao uso de tecnologias não invasivas de cuidado como estratégias na desmedicalização do parto. Esc Anna Nery Rev Enferm 2004; 8(2):194-7. 
27. Costa AF. Óleos essenciais na gestação, parto e pós-parto.[Internet] Instituto Brasileiro de Aromatologia/Laszlo. Belo Horizonte, 2014. Disponível em: http://laszlo.ind.br/campanhas/OLEOS_ESSENCIAIS_NA_GESTA\%C3\%87\%C3\%83O_Andre _Ferraz.pdf

28. Gnatta JR, Kurabayashi LFS, Turrini RNT, Silva MJP. Aromatherapy and nursing: historical and theoretical conception. Revista da Escola de Enfermagem da USP 2016;50(1):127-33.

29. Conselho Federal de Enfermagem. Resolução COFEN 197/1997. Estabelece e reconhece as Terapias Alternativas como especialidade e/ou qualificação do profissional de Enfermagem [Internet]. Brasília; 1997. Disponível em: http:// www.cofen.gov.br/resoluo-cofen-1971997_4253.html

30. Dhany AL, Mitchell T, Foy C. Aromatherapy and massage intrapartum service upon use of analgesia and anesthesia in women in labour; a retrospective case note analysis. J Altern Complement Med 2012; 18(10):932-8.

31. Jones L, Othman M, Dowswell T, Alfirevic Z, Gates S, Newburn M et al. Pain management for women in labour: an overview of systematic reviews. Cochrane Database Syst Rev 2012; 14(3):CD009234.

32. Hosseni SF, Pilevarzadeh M, Vazirinasab H. Non-pharmacological strategies on pain relief during labor. BiosciBiotech Res Asia 2016;13(2):701-6.

33. Angel RG, Sriram N, Soli TK. Complementary and alternative approaches to pain relief during labor.Int J of Allied MedSci and Clin Research 2013;1(2):65-9.

34. Burns E, Zobbi V, Panzeri D, Oskrochi R, Regalia A. Aromatherapy in childbirth: a pilot randomised controlled trial. BJOG Na Int J Obstet Gynaecol 2007;114(7):838-44.

35. Gayeski ME, Brüggmann OM. Métodos não farmacológicos para alívio da dor no trabalho de parto: uma revisão sistemática. Texto\&Contexto Enferm 2010; 19(4):774-82.

36. Arendt KW, Tessmer-Tuck JA. Nonpharmacologic labor analgesia. ClinP erinatol 2013; 40(3):351-71.

37. Vakilian K, Karamat A, Mousavi A, Shariati M, Ajami E, Atarha M. The effect of Lavender essence via inhalation method on labor pain. J Shahrekord Univ Med Sci 2012;14(1):34-40.

38. Alavi N, Nemati M, Kaviani M, Tabaie M. The effect of lavender aromatherapy on the pain intensity perception and intrapartum outcomes in primiparous. Armaghanedanesh Journal 2010;15(1):30-7.

39. Seraji A, Vakilian K. The comparison between the effects of aromatherapy with lavender and breathing techniques on the reduction of labor pain. Nurs Midwifery Stud 2013;2(1):115-9.

40. Abbaspoor Z, Mohammadkhani Shari L. Lavender aromatherapy massages in reducing labor pain and duration of labor: a randomized controlled trial. African J Pharm Pharmacol 2013;7(8):456-30.

41. Burns EE, Blamey C, Ersser SJ, Barnetson L, Lloyd AJ. An investigation into the use of aromatherapy in intrapartum midwifery practice. J Altern Complement Med 2000;6(2):141-7.

42. MohammadkhaniShari L, Abbaspoor Z, Aghel N, MohammadkhaniShari S. Effect of massage aromatherapy with lavender oil on pain intensity of active phase of labor in nulliparous women. J Med Plants 2012;2(42):167-76.

43 Makvandi S, Mirteimoori M, Najmabadi KM, et al. A review of randomized clinical trials on the effect of aromatherapy with lavender on labor pain relief. Nurse Care Open Acces J 2016;1(3):42-47.

44. Yazdkhasti M; Pirak A. The effect of aromatherapy with lavender essence on severityof labor pain and duration of labor in primiparous women. Complementary Therapies in Clinical Practice 2016; 25: 81-86. 